\title{
BMJ Open Rationale and design of TransplantLines: a prospective cohort study and biobank of solid organ transplant recipients
}

\author{
Michele F Eisenga, ${ }^{1}$ Antonio W Gomes-Neto, ${ }^{1}$ Marco van Londen, ${ }^{1}$ \\ Aaltje L Ziengs, ${ }^{1,2}$ Rianne M Douwes, ${ }^{1}$ Suzanne P Stam, ${ }^{1}$ Maryse C J Osté, ${ }^{1}$ \\ Tim J Knobbe, ${ }^{1}$ Niek R Hessels, ${ }^{1}$ Anne M Buunk, ${ }^{2}$ Coby Annema, ${ }^{3}$ \\ Marion J Siebelink, ${ }^{3}$ Emoke Racz, ${ }^{4}$ Jacoba M Spikman, ${ }^{2}$ Frank A J A Bodewes, ${ }^{5}$ \\ Robert A Pol, ${ }^{6}{ }^{\text {Stefan } P}$ Berger, ${ }^{1}$ Gea Drost, ${ }^{7}$ Robert J Porte, ${ }^{6}$ \\ Henri G D Leuvenink, ${ }^{6}$ Kevin Damman, ${ }^{8}$ Erik A M Verschuuren, ${ }^{9}$ \\ Vincent E de Meijer, ${ }^{6}$ Hans Blokzijl, ${ }^{10}$ Stephan J L Bakker ${ }^{1}$
}

To cite: Eisenga MF, GomesNeto AW, van Londen M, et al. Rationale and design of TransplantLines: a prospective cohort study and biobank of solid organ transplant recipients. BMJ Open 2018;8:e024502. doi:10.1136/ bmjopen-2018-024502

- Prepublication history for this paper is available online. To view these files please visit the journal online (http://dx.doi. org/10.1136/bmjopen-2018024502).

MFE, AWG-N and ML contributed equally.

Received 29 May 2018 Revised 19 September 2018 Accepted 25 October 2018

Check for updates

(c) Author(s) (or their employer(s)) 2018. Re-use permitted under CC BY-NC. No commercial re-use. See rights and permissions. Published by BMJ.

For numbered affiliations see end of article.

Correspondence to

Michele F Eisenga;

m.f.eisenga@umcg.nl

\section{ABSTRACT}

Introduction In the past decades, short-term results after solid organ transplantation have markedly improved. Disappointingly, this has not been accompanied by parallel improvements in long-term outcomes after transplantation. To improve graft and recipient outcomes, identification of potentially modifiable risk factors and development of biomarkers are required. We provide the rationale and design of a large prospective cohort study of solid organ transplant recipients (TransplantLines).

Methods and analysis TransplantLines is designed as a single-centre, prospective cohort study and biobank including all different types of solid organ transplant recipients as well as living organ donors. Data will be collected from transplant candidates before transplantation, during transplantation, at 3 months, 6 months, 1 year, 2 years and 5 years, and subsequently every 5 years after transplantation. Data from living organ donors will be collected before donation, during donation, at 3 months, 1 year and 5 years after donation, and subsequently every 5 years. The primary outcomes are mortality and graft failure. The secondary outcomes will be cause-specific mortality, cause-specific graft failure and rejection. The tertiary outcomes will be other health problems, including diabetes, obesity, hypertension, hypercholesterolaemia and cardiovascular disease, and disturbances that relate to quality of life, that is, physical and psychological functioning, including quality of sleep, and neurological problems such as tremor and polyneuropathy.

Ethics and dissemination Ethical approval has been obtained from the relevant local ethics committee. The TransplantLines cohort study is designed to deliver pioneering insights into transplantation and donation outcomes. The study design allows comprehensive data collection on perioperative care, nutrition, social and psychological functioning, and biochemical parameters. This may provide a rationale for future intervention strategies to more individualised, patient-centred transplant care and individualisation of treatment. Trial registration number NCT03272841.

\section{Strengths and limitations of this study}

- This is a large biobank and cohort study with extensive data collection on a myriad topics related to transplantation and/or donation.

- The study included all types of solid organ transplant recipients.

- The study has a long follow-up to assess many relevant clinical outcomes.

- This is a single-centre study.

- Residual confounding cannot be excluded due to observational design.

\section{BACKGROUND}

Solid organ transplantation is the preferred treatment for end-stage organ failure. During the past decades, advances in immunosuppressant medications, treatment of infections, perioperative medical care and surgical techniques (including living donation) have led to important improvements in early post-transplant graft and patient survival. ${ }^{1}$ However, in the long term, graft failure is a major cause of patient mortality and morbidity in all types of transplantation..$^{2-4}$ For example, in renal transplant recipients, half of the cadaveric renal allografts fail within a timeframe of 10 years. ${ }^{5}$ Apart from reduced survival, transplant recipients often develop health problems that greatly reduce their perceived quality of life (figure 1).$^{6-8}$

The multitude of health problems that recipients experience after transplantation includes, among others, obesity, diabetes, hypertension, heart failure and malignancies. $^{9-11}$ These are likely the consequence of a combination of factors, including (1) continuous exposure to treatment with immunosuppressive drugs necessary for prevention of 
Patient and Graft survival

(e.g. poor long-term outcomes)

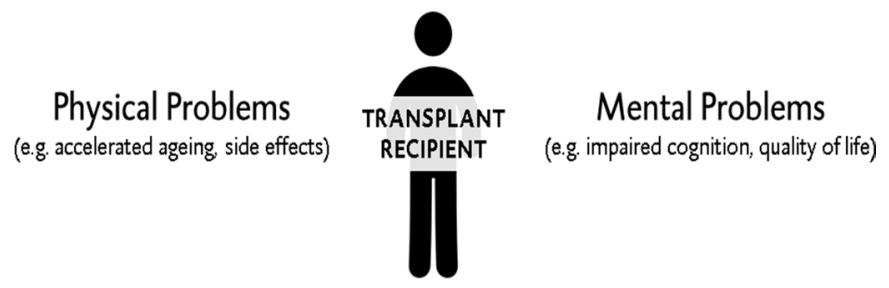

\section{Social Problems}

(e.g. participation loss, poor coping)

Figure 1 Overview of different health problems that arise in the long term after transplantation, physical, psychological and social.

rejection of the transplanted organ, (2) damage induced by pre-existing exposure to end-stage organ failure, and (3) adverse lifestyle and environmental factors, all potentially expressed against (4) a background of increased (epi)genetic susceptibility. Among these, immunosuppressive treatment, adverse lifestyle and environmental factors are good candidates for modification to decrease the load of post-transplant health problems. It should be realised that immunosuppressive treatment is currently mainly 'one-size fits all'. Hence, improvement can be achieved by the development of biomarkers that can allow for recognition of transplant recipients in which immunosuppressive load can be safely reduced or in which certain drugs can better be avoided, and of biomarkers which can guide such individualised immunosuppressive treatment. To improve long-term transplant outcomes, it is imperative to identify modifiable risk factors, especially among those recipients who are at increased risk.

To date, it is largely unknown in which transplant recipients immunosuppressive medication can be safely reduced to prevent the development of health problems. Furthermore, in terms of healthcare costs, it is important to prevent recurrent hospital admissions, retransplantations or-in the case of kidney transplantation-return to dialysis, which are all associated with very high expenses. ${ }^{12}$ To effectively develop interventions to reduce mortality and morbidity after transplantation, more research is necessary on clinical and biochemical risk factors present in transplant recipients. Also, the use of living donors for kidney and liver transplantation requires a living donor programme with good long-term outcomes for the donor and the recipient. Living kidney donors, for example, have an increased risk of end-stage renal disease, ${ }^{3} 14$ while only registry data exist on the effect of living donor characteristics on recipient outcomes. ${ }^{15} 16$

Until now, many registries and large cohort studies focus on one type of solid organ transplantation, limiting comparability between different transplant populations. As a result, studies investigating biomarkers, quality of life, and the development of health problems and adverse outcomes across different solid organ transplant populations are scarce. Despite the differences which exist in patient characteristics and treatment after different solid organ transplantations, there are many similarities in health problems that occur among subtypes of transplantation. The objective of the TransplantLines study is to identify the risk factors for the development of long-term health problems after transplantation and to develop new interventions to improve outcome, both combined for all solid organ transplant recipients as well as specific for each subtype of transplantation.

\section{METHODS/DESIGN}

\section{Study design and setting}

The TransplantLines study is a unique, novel prospective biobank and cohort study which aims to provide a better understanding of the causes of disease-related and ageing-related outcomes and health problems, both physical and psychological, in solid organ transplant recipients and donors. The University Medical Center Groningen (UMCG) is the largest transplantation centre in the Netherlands, and the only Dutch centre that covers all types of solid organ transplantation, as well as living kidney and liver donation programmes. All participants will give written informed consent on enrolment. Follow-up and prospective events will be recorded over time. An overall participation rate of $85 \%$ is expected across the different transplant populations and a total number of 3000 participants is aimed.

\section{Transplant patients}

The study population comprises all solid organ transplant recipients, that is, heart, lung, kidney, liver and small bowel transplant recipients. Both new transplant candidates as well as transplant recipients are eligible to participate in the study. Participants of all ages will be included in TransplantLines. Children (age $<18$ years) will be eligible for participation on consent by a legal representative $(<12$ years) or a shared consent of both the child and the legal representative ( $\geq 12$ years). The study will also include candidates for retransplantation. Exclusion criteria for participation in the TransplantLines study will be no mastery of the Dutch language or no capability to intellectually comprehend questionnaires or physical tests.

\section{Living donors}

Living kidney and liver donors will also be included in the study. The goal of including donors is to study the effects of donation and improve living donor safety, and donors will serve as controls for their recipients, allowing for matched longitudinal analyses. Prospective living kidney and liver donor candidates ( $\geq 18$ years old) will be eligible to participate in the study, as well as living organ donors who have donated an organ prior to the start of the TransplantLines study. Exclusion criteria will be no mastery of the Dutch language or no capability to intellectually comprehend questionnaires or physical tests. 


\section{Transplant Recipients}

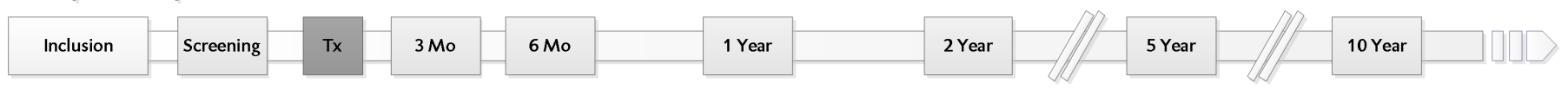

\section{Living Donors}

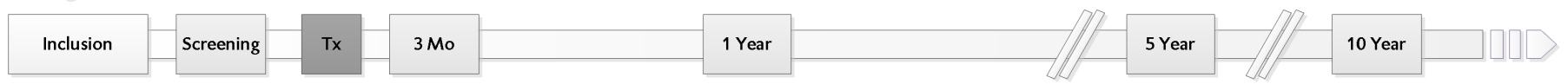

Figure 2 Flow chart of the different visits in the TransplantLines study. At every study visit, biobank, general tests and questionnaires will be performed. Specifically addition at each timepoints; at time of transplantation, perioperative residual material will be collected. At 3 months after transplantation, cognitive protocol will be performed. At 6 months, physical protocol will be carried out. At 12 months, randomisation to physical or cognitive protocol will occur. At 2 years after transplantation, a limited set of tests will be executed. Follow-up will be performed every 5 years.

\section{Transplant recipients timeline}

All participants of the TransplantLines study will be examined at fixed timepoints, as shown in figure 2. Transplant candidates will be first seen at pretransplant screening. Prior to transplantation, all transplant candidates undergo routine clinical screening. Generally, transplant candidates will be transplanted if surgery risks and transplant benefit are optimised, based on an individualised multidisciplinary clinical decision. Further study visits will be performed at the time of transplantation, at 3 months, 6 months, 12 months and 2 years after transplantation, and hereafter follow-up will be performed at 5 years after transplantation and every consecutive 5 years. At time of transplantation means during operation prior to surgical incision. At that timepoint, the blood samples are being drawn by the anaesthesiologists taking care of the patient. The difference with this sample compared with other previous samples is that this sample is taken during the operation, whereas the other samples are not. For example, a kidney transplant candidate can be screened and included in the TransplantLines study, but may need to wait 2 years on the waiting list prior to receiving the actual transplantation. Since we realise that a study of this size and duration combined with the frequency of study visits will result in lower subject adherence, we estimate a $10 \%$ dropout overall in follow-up in this group of transplant candidates.

Transplant recipients with a functional graft for at least 1 year post-transplantation and who received a solid organ transplant prior to the start of the TransplantLines study will be included at the next outpatient clinic visit. Henceforth, patients will be examined every 5 years and follow-up samples will be collected. Aside from the fixed timepoints, biobank samples of transplant recipients will be collected at the time of protocol biopsies that are performed in the kidney transplant programme (6 months after transplantation) and the heart transplant programme (repeatedly during the first year after transplantation), and if a biopsy is taken, on clinical indication, usually because of worsening of transplant function, with suspicion of acute or chronic rejection.

If a subject gets retransplanted with the same kind of organ, this will be classified as graft failure and the end of follow-up. Subjects will not be included in the primary database twice. Yet we will allow for inclusion of subjects retransplanted with the same kind of organ with a new identification in the transplant candidate group, but this will be with the intention to build over time a separate cohort with data and a biobank on retransplantations. When a transplant recipient is later on transplanted with another kind of organ, follow-up will be for the initially transplanted organ. Transplant recipients receiving a combined transplantation, for example, kidney-pancreas and kidney-liver, will be treated as separate groups, not to be included in overall analyses for the much larger groups of subjects with single transplanted organs.

In case a transplant recipient moves to another region of the Netherlands or abroad, the transplant recipient will always require continued medical care and follow-up by a medical specialist, who will require thorough medical information on the patient and the transplanted organ, to allow for continued dedicated care. Therefore, the medical specialist who will continue care will seek contact for information, and it will usually be possible to continue follow-up on long-term outcome and events via this medical specialist. So follow-up is usually assured and loss to follow-up will be rare. Since study visits are combined with routine clinical visits, subjects who move out of our region will be excluded from further study visits for the TransplantLines study.

\section{Living donors timeline}

All donors of the TransplantLines study will be examined at fixed timepoints, as shown in figure 2 . The first study visit of donor candidates will occur at predonation screening. Prior to donation, all candidates undergo routine clinical screening. Generally, donors will be accepted if surgery risks and transplant benefit are optimised, based on an individualised multidisciplinary clinical decision taking national and international guidelines into account. ${ }^{17} 18$ Subsequently, study visits will be performed at the time of nephrectomy and at 3 months postdonation. At 12 months postdonation, donors will fill in a questionnaire, and at 5 and 10 years postdonation there will be another study visit. Hereafter follow-up will be performed every 5 years. Living organ donors who have donated an organ prior to the start of the TransplantLines study will be included at their next donor follow-up visit to their outpatient clinic. 


\section{Patient and public involvement}

The aim of the TransplantLines study is to provide a better understanding of the causes of disease-related and ageing-related outcomes and health problems. This aim was derived from patient surveys, and parts of the collected data are codesigned by patients and healthcare professionals. Because of its scope, patients will play a role in the organisation of the study, helping with recruitment and conduct of the study. Also, students from a broad range of studies will play a role in the organisation of the study, for example, master students from medicine, biomedical sciences, neuropsychology, psychology, physical therapy, communication sciences, dietitian students and laboratory technician students. Patients and collaborators will be informed of major study results by press releases from the UMCG.

\section{Data collection}

\section{Biobank}

Blood, 24-hour urine, faeces, nails and hair will be collected from the participants at each TransplantLines visit. Participants will be instructed to collect a 24-hour urine sample according to strict protocol on the day before their visit to the outpatient clinic, that is, discard their morning urine specimen, collect all subsequent urine throughout the next 24 hours and include the next morning's first specimen of the day of the visit to the outpatient clinic. Blood will be drawn after an overnight fasting period of 8-12 hours in the morning after completion of the 24-hour urine collection. Blood drawing and receipt of the collected 24-hour urine samples are performed by experienced nurses at our outpatient clinic.

As blood samples, one serum tube of $10 \mathrm{~mL}$, two EDTA samples of $10 \mathrm{~mL}$, one citrate tube of $6 \mathrm{~mL}$, one lithium-heparin tube of $10 \mathrm{~mL}$ and one PAXgene tube of $10 \mathrm{~mL}$ will be collected from each participant at each TransplantLines visit. Subsequently, tubes will be centrifuged by technicians at $1300 \mathrm{~g}$ for $10 \mathrm{~min}$, except for the citrate tube which is centrifuged at $2500 \mathrm{~g}$ for $10 \mathrm{~min}$. Of the 24-hour urine collection, three urine tubes will be collected, of which one tube will be partially acidified. All blood and urine samples will be subsequently aliquoted by technicians and shipped to the core laboratory for storage in $-80^{\circ} \mathrm{C}\left(-112^{\circ} \mathrm{F}\right)$ freezers (Panasonic, 's-Hertogenbosch, The Netherlands) (table 1). Blood and urine samples will be analysed in the following years for multiple research questions that will arise.

Participants will be asked to collect a faeces sample the day prior to the TransplantLines visit. A FecesCatcher (TAG Hemi VOF, Zeijen, The Netherlands) will be sent to the patient's home, and faeces sample will be collected in appropriate tubes and frozen immediately after collection. The participant will transport the faeces sample in cold storage (with ice cubes or in a cooler) to the TransplantLines visit the following day. Subsequently, the faeces sample will be immediately stored at $-80^{\circ} \mathrm{C}\left(-112^{\circ} \mathrm{F}\right)$. Faeces samples will be primarily used for microbiome analyses. Solid organ transplant recipients have a shift

\begin{tabular}{|c|c|c|c|c|}
\hline Sample & Colour code & Tube size & $\mathbf{n}$ & Temperature \\
\hline Serum & Red & $1500 \mu \mathrm{L}$ & 4 & $-80^{\circ} \mathrm{C} /-112^{\circ} \mathrm{F}$ \\
\hline $\begin{array}{l}\text { EDTA } \\
\text { plasma }\end{array}$ & Purple & $1500 \mu \mathrm{L}$ & 6 & $-80^{\circ} \mathrm{C} /-112^{\circ} \mathrm{F}$ \\
\hline Buffy coat & Purple & NA & 1 & $-80^{\circ} \mathrm{C} /-112^{\circ} \mathrm{F}$ \\
\hline $\begin{array}{l}\text { Blood with } \\
\text { RBC }\end{array}$ & Purple & $1500 \mu \mathrm{L}$ & 2 & $-80^{\circ} \mathrm{C} /-112^{\circ} \mathrm{F}$ \\
\hline $\begin{array}{l}\text { Lithium- } \\
\text { heparin }\end{array}$ & Green & $1500 \mu \mathrm{L}$ & 4 & $-80^{\circ} \mathrm{C} /-112^{\circ} \mathrm{F}$ \\
\hline Citrate & Blue & $500 \mu \mathrm{L}$ & 4 & $-80^{\circ} \mathrm{C} /-112^{\circ} \mathrm{F}$ \\
\hline PAXgene & Transparent & $2.5 \mathrm{~mL}$ & 1 & $-80^{\circ} \mathrm{C} /-112^{\circ} \mathrm{F}$ \\
\hline $\begin{array}{l}\text { 24-hour } \\
\text { urine }\end{array}$ & Yellow & $1500 \mu \mathrm{L}$ & 6 & $-80^{\circ} \mathrm{C} /-112^{\circ} \mathrm{F}$ \\
\hline $\begin{array}{l}\text { Acidified } \\
\text { 24-hour } \\
\text { urine }\end{array}$ & Yellow & $2000 \mu \mathrm{L}$ & 2 & $-80^{\circ} \mathrm{C} /-112^{\circ} \mathrm{F}$ \\
\hline Faeces & Black & $20 \mathrm{~mL}$ & 1 & $-80^{\circ} \mathrm{C} /-112^{\circ} \mathrm{P}$ \\
\hline Nails & Purple & $0.5 \mu \mathrm{L}$ & 1 & $-80^{\circ} \mathrm{C} /-112^{\circ} \mathrm{F}$ \\
\hline Hair & Purple & $2000 \mu \mathrm{L}$ & 1 & $-80^{\circ} \mathrm{C} /-112^{\circ} \mathrm{F}$ \\
\hline
\end{tabular}

NA, not applicable; RBC, red blood cell.

in the gut microbiome with a decrease in predominant organisms, a loss of bacterial diversity and emergence of new dominant population. These may result in increased risk of infection, rejection and mortality. Therefore, we would like to examine the gut microbiome in relation to the development of health problems after transplantation.

Additional blood and urine samples will also be collected in the event of worsening graft function and an organ transplant biopsy is indicated. Prior to the biopsy, one serum tube of $10 \mathrm{~mL}$, two EDTA samples of $10 \mathrm{~mL}$, one citrate tube of $6 \mathrm{~mL}$, one lithium-heparin tube of $10 \mathrm{~mL}$ and one PAXgene tube of $10 \mathrm{~mL}$ will be collected. At the same time, one serum tube of $10 \mathrm{~mL}$, one EDTA sample of $10 \mathrm{~mL}$ and one spot urine sample of $10 \mathrm{~mL}$ will be collected and directly stored on ice to prevent (ongoing) in vitro complement activation.

Furthermore, during transplant surgery and transplant biopsies, tissue samples will be collected of the transplanted organ and surrounding tissues, including fat, skin, ureter, tracheal, biliary, and arterial and venous tissues, that have been discarded as pathological waste.

\section{Clinical and laboratory characteristics}

Clinical laboratory measurements requested by the physician will be included in the study database on patient consent. Most study visits are at the outpatient clinic, and for these visits blood samples will be taken fasting in the morning. It is unlikely that at these study visits multiple labs will be obtained on the same day, but if they are taken only the lab obtained at the time of the study visit will be included in the database. In the likely rare case that multiple labs are taken on the day of a study visit, this will 


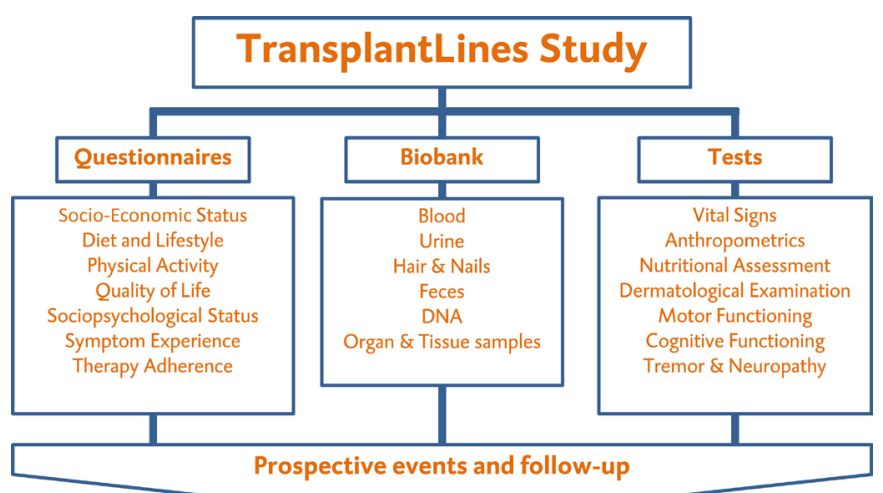

Figure 3 Overview of the three main pillars of the TransplantLines study, that is, questionnaires, biobank and tests. The collection of data in these pillars at multiple timepoints will allow investigation of whether biomarkers at baseline can better predict the occurrence of adverse outcomes and whether correction could possibly result in an improved survival.

likely be a sign of an acute event that occurred after the study visit and it will then later on be linked to the database as the event that occurred. At the visit for transplant surgery, multiple labs will be obtained on the same day. On that day, only the lab results available from the samples which are taken at the same time of sampling during surgery to provide for samples that will be included in the biobank will be linked to the database. These lab results are recognisable by the routine assays that are performed, because they are more extensive and include other routine lab results than the routine lab results available from the samples taken at other times on the same day. Demographic characteristics along with data on medication use will be provided by the participants and will be verified using electronic hospital records. Medical information including donor and recipient information at the time of transplantation, underlying disease, hospital admissions, complications after transplantation, further surgical or other interventional treatments, comorbidities, graft failure, and mortality will be extracted from electronic hospital records.

\section{Questionnaires}

Biobank data will be expanded with an extensive set of questionnaires to collect data on physical, psychological and social impact of undergoing a transplantation (figure 3). Transplant candidates will be asked to fill out a comprehensive questionnaire during screening prior to transplantation and at 1-year post-transplantation. Transplant recipients with a functional graft for more than 1 year post-transplantation and who received the solid organ prior to the start of the TransplantLines study will be asked to complete the same questionnaire. A subset of questionnaires will be provided at other predefined timepoints, that is, at 3 , at 6 months and at 2 years after transplantation. Topics addressed by questionnaires include, among others, nutritional intake and diet, health-related quality of life, lifestyle factors such as physical activity,
Table 2 List of questionnaires in the TransplantLines study

\begin{tabular}{|c|c|}
\hline Questionnaires & Related subject \\
\hline $\mathrm{EQ}^{6} \mathrm{D}^{60}$ & EuroQoL six dimensions \\
\hline VAS $^{61}$ & Visual Analogue Scale \\
\hline SF36 62 & Short Form-36 Health Survey \\
\hline SQUASH ${ }^{63}$ & $\begin{array}{l}\text { Short Questionnaire to Assess Health- } \\
\text { Enhancing Physical Activity }\end{array}$ \\
\hline BAASIS $^{64}$ & $\begin{array}{l}\text { Basel Assessment of Adherence to } \\
\text { Immunosuppressive Medications Scale }\end{array}$ \\
\hline MTSOSDS-R5965 & $\begin{array}{l}\text { Modified Transplant Symptom } \\
\text { Occurrence and Symptom Distress scale }\end{array}$ \\
\hline $\mathrm{CIS}^{66}$ & Checklist Individual Strength (Fatigue) \\
\hline$P S Q I^{67}$ & Pittsburgh Sleep Quality Index \\
\hline STAI6 $6^{68}$ & Short form State Trait Anxiety Inventory \\
\hline PHQ969 & Patient Health Questionnaire (Depression) \\
\hline $\mathrm{CFQ}^{70}$ & Cognitive Functioning Questionnaire \\
\hline WHO- $5^{71}$ & $\begin{array}{l}\text { World Health Organization-5 (Well-Being } \\
\text { Index) }\end{array}$ \\
\hline
\end{tabular}

\begin{tabular}{|c|c|}
\hline $\mathrm{TxEQ}^{72}$ & Transplant Effects Questionnaire \\
\hline Mastery Scale ${ }^{73}$ & Pearlin Mastery Scale \\
\hline UCL-47 & Utrecht Coping List- 47 \\
\hline USER-P & $\begin{array}{l}\text { Utrecht Scale for Evaluation of } \\
\text { Revalidation-Participation }\end{array}$ \\
\hline Work & Participation in Labour \\
\hline WRFQ $^{74}$ & Work Role Functioning Questionnaire \\
\hline FAD & Family Assessment Device \\
\hline $\mathrm{ABO}$ & $\begin{array}{l}\text { Active Engagement, Protective Buffering } \\
\text { and Overprotection Questionnaire }\end{array}$ \\
\hline $\begin{array}{l}\text { Social Support } \\
\text { Questionnaire }\end{array}$ & Social support \\
\hline $\mathrm{DAG}^{75}$ & $\begin{array}{l}\text { DArmGezondheid (Bowel Health) } \\
\text { Questionnaire }\end{array}$ \\
\hline $\mathrm{FFQ}^{76}$ & Food Frequency Questionnaire \\
\hline $\begin{array}{l}\text { LIVAS Scale for } \\
\text { Physical Self- } \\
\text { Efficacy movement }\end{array}$ & $\begin{array}{l}\text { Lichamelijke vaardigheden schaal, Dutch } \\
\text { version of the Perceived Physical Activity } \\
\text { Scale }\end{array}$ \\
\hline $\begin{array}{l}\text { OBiN Sedentary } \\
\text { Behaviour } \\
\text { Questionnaire }\end{array}$ & Sedentary behaviour \\
\hline
\end{tabular}

Smoking Behaviour Smoking behaviour

Questionnaire

Alcohol Use Alcohol use

Disorders

Identification Test

(AUDIT)*

${ }^{*}$ The AUDIT questionnaire will only be gathered from liver transplant recipients.

sleep quality and smoking behaviour, psychological impact such as anxiety, depression, coping and well-being, and social impact such as employment and family relationships. Specification of all the different questionnaires with related subject is shown in table 2. Questionnaires will be sent digitally or by mail, as requested. During study 
visits, all questionnaires will be checked by a trained investigator for completeness and validity.

\section{Standard assessments}

Blood pressure $(\mathrm{mm} \mathrm{Hg}$ ) will be measured according to a standard clinical protocol using an automatic device (Philips Suresign VS2 ${ }^{+}$, Andover, Massachusetts, USA). To prevent a white-coat effect, participants will be seated during which blood pressure and heart rate will be measured four times, with an interval of 3 min between measurements. Hereafter, participants will be asked to stand up straight for $1 \mathrm{~min}$, after which blood pressure and heart rate measurements will be repeated once in standing position. Measurements will be performed with participants being on their regular medication, including antihypertensive drugs at trough.

Anthropometry measurements will include body weight, body length, and waist and hip circumference. Body weight $(\mathrm{kg})$ will be measured in lightweight clothing without shoes using a calibrated digital measuring scale (seca 877, seca, Hamburg, Germany). Height $(\mathrm{cm})$ will be measured using a wall-secured stadiometer (seca 222). Waist and hip circumference $(\mathrm{cm})$ will be calculated using a measuring tape roll with standardised retraction mechanism (seca 201). Waist circumference will be measured mid-way between the lowest rib and the iliac crest with the participant in standing position. Hip circumference will be determined at the maximum circumference over the trochanter major. All anthropometry measurements will be assessed twice, with inclusion of a third measurement contingent on a difference of more than half a kilogram in weight or more than $1 \mathrm{~cm}$ in length.

Hand grip strength will be assessed with the Jamar Hydraulic Hand Dynamometer (Patterson Medical JAMAR 5030J1, Warrenville, Canada). ${ }^{19}$ Participants will be instructed to sit in a chair with their shoulders in adduction, their arms rotated into neutral position, their elbows flexed to $90^{\circ}$, and forearms and wrists held in neutral position. Hereafter, participants will be instructed to perform a maximal isometric contraction. Hand grip strength will be tested three times with an interval of $30 \mathrm{~s}$ rest for recovery between each attempt. The dominant hand will be stated in all measurements. Furthermore, to create uniformity among assessments, the second handle position of the hand dynamometer will be used, which has been shown to be the most accurate position. ${ }^{20}$

Lung function will be measured by means of an Asma-1 handheld spirometer (Vitalograph, Buckingham, UK) ${ }^{21}$ Of all participants, forced expiratory volume in $1 \mathrm{~s}$, as a marker of lung function, will be recorded.

Body composition will be determined using a multifrequency bioelectrical impedance device (BIA, Quadscan 4000, Bodystat, Douglas, British Isles) at 5, 50, 100 and $200 \mathrm{~Hz}$, which allows to distinguish between lean body mass and fat body mass taking into account differences in volume status. ${ }^{22}$ The main outcome variables from the BIA are estimated fat mass, fat-free mass and body fat percentage. In brief, the BIA measurement will be performed with the participant in supine position with arms and legs abducted from the body. Sensor electrodes will be placed on the dorsum of the right hand and feet, with a minimal distance of $5 \mathrm{~cm}$ between the electrodes. Measurement will not be executed if the participant has a temperature exceeding $37.9^{\circ} \mathrm{C} / 100.2^{\circ} \mathrm{F}$ or has a functioning implantable cardioverter-defibrillator/ pacemaker.

Advanced glycation endproducts (AGEs) will be determined using an AGE Reader SU (DiagnOptics Technologies, Groningen, The Netherlands).$^{23}$ The AGE Reader SU measures skin autofluorescence using the characteristic fluorescent properties of certain AGEs to estimate the level of AGE accumulation in the skin. AGEs have been implicated in the pathogenesis of vascular damage and cardiovascular disorders and aid in characterising the cardiovascular risk profile of transplant recipients. ${ }^{24}$

Transplant recipients are known to be at increased risk for cutaneous malignancies, mainly related to longterm use of immunosuppressive medication. ${ }^{25}$ To identify which transplant recipients are especially prone to develop dermatological health problems, a detailed dermatological history with emphasis on malignancies and subsequent treatment will be obtained. Next, a standardised dermatological examination will be performed by the trained investigator. The dermatological examination includes the determination of eye colour, natural hair colour at adolescence and skin type according to the classification of Fitzpatrick. ${ }^{26}$ In addition, the presence and quantity of lentigines, moles, freckles and warts are examined.

To assess frailty, the Clinical Frailty Scale (CFS) will be scored at study visits by the trained investigator. The CFS is a validated frailty measurement, and frailty is scored based on clinical judgement on a continuous scale from 1 (very fit) to 9 (terminally ill). A CFS score of $\geq 5$ is generally considered frail. ${ }^{27}$

To assess nutritional status, a Patient-Generated Subjective Global Assessment (PG-SGA, PT-Global, Philadelphia, USA) will be scored..$^{27} 28$ The PG-SGA is a patient-centred adaptation of the original Subjective Global Assessment. The different domains assessed by the PG-SGA are (1) changes in body weight, (2) changes in nutritional intake, (3) symptoms which negatively influence intake, absorption and utilisation of nutrients, (4) level of activities and function, (5) conditions that increase nutritional risk or requirements, (6) metabolic stress, and (7) physical examination. Based on the PG-SGA score, subjects can be classified as well-nourished, moderately malnourished or severely malnourished.

\section{Randomisation and additional physical and cognitive tests}

In addition to standard assessments, participants will receive additional physical tests or cognitive tests at their study visit at 12 months post-transplantation or at the first study visit if it concerns transplant recipients with a functioning graft for more than 1 year who were transplanted before the start of TransplantLines. 
Participants will be randomised (1:1 ratio) into either the 'physical' arm or the 'cognitive' arm of the study. Randomisation will be performed for each transplant programme separately to ensure balanced randomisation of subjects for each type of solid organ transplant. Participants randomised into the 'physical' arm of the study protocol will be asked to accomplish a standing balance test, a 2-Minute Walk Test (2MWT), a 4-Metre Walk Test (4MWT), a dexterity test, a Five Time Sit To Stand (FTSTS) test, Timed Up and Go (TUG) test, a rigorous neurological examination and a breath analysis. With inclusion of the first four tests together with the hand grip strength, the five physical components of the National Health Institute Toolbox for motor assessment are being assessed. ${ }^{29}$ A subset of these tests will also be performed at 6 months post-transplantation study visit in all solid organ transplant recipients. Participants randomised into the 'cognitive' arm of the study protocol undergo a series of neuropsychological tests performed by a trained neuropsychologist or master student neuropsychology under the supervision of a trained neuropsychologist. The tests are administered in a quiet room with no disturbances. For timed tests, a digital clock is used. The tests are performed in a fixed order and no feedback regarding the results is given to the participant during administration. An overview of the different tests performed in TransplantLines including the neuropsychological tests is specified in table 3. A subset of the neuropsychological tests will also be performed at 3 months

Table 3 Overview of the different tests performed in TransplantLines study per study protocol

\begin{tabular}{|c|c|}
\hline Parameter/Test & Details \\
\hline \multicolumn{2}{|l|}{ General (all protocols) } \\
\hline General parameters & $\begin{array}{l}\text { Collection of Biobank material and evaluation of questionnaires, check quality of } \\
\text { data }\end{array}$ \\
\hline Blood pressure & Using an automatic or semiautomatic device \\
\hline Weight & Using digital measuring scale \\
\hline Length & Using measuring tape fixed to the wall \\
\hline Waist and hip size & Using measuring tape roll \\
\hline BIA & Bioimpedance analysis (Quadscan 4000) \\
\hline SAF & Skin autofluorescence (AGE Reader SU) \\
\hline Dermatological questionnaire & After physical examination by student researcher \\
\hline Clinical Frailty Scale & After physical examination by student researcher \\
\hline PG-SGA & Scored Patient-Generated Subjective Global Assessment \\
\hline Lung function & Using spirometry (Vitalograph Asma-1) \\
\hline Breath analysis & Using QuinTron BreathTracker \\
\hline \multicolumn{2}{|l|}{ Physical protocol } \\
\hline Balance test & Using Axivity accelerometer \\
\hline Hand grip & Using hydraulic handheld dynamometer \\
\hline Physical strength & Multiple muscle groups, using digital dynamometer \\
\hline Sensibility tests & Using pin-prick, monofilament and biothesiometer \\
\hline Tremor analysis & Using Tetras scale and Axivity accelerometers \\
\hline Manual dexterity & Using dexterity PEG-Board \\
\hline \multicolumn{2}{|l|}{ Cognitive protocol } \\
\hline Nederlandse Leestest voor Volwassenen & Dutch version of the National Adult Reading Test \\
\hline Digit Span & Subtest of the Wechsler Adult Intelligence Scale IV \\
\hline 15 Words Test & Dutch version of Ray Auditory Verbal Learning Test \\
\hline Cognitive Screening Test & Cognitive Screening Test (20) \\
\hline \multicolumn{2}{|l|}{ Trail Making Test } \\
\hline \multicolumn{2}{|l|}{ Clock-Drawing Test } \\
\hline \multicolumn{2}{|l|}{ Symbol Digit Modalities Test } \\
\hline Letter Fluency Test & Dutch version of the Controlled Word Association Test \\
\hline Word Fluency Test & Subtest of the Groningen Intelligence Test \\
\hline Key Search Test & Subtest of the Behavioural Assessment of the Dysexecutive Syndrome \\
\hline
\end{tabular}


post-transplantation in all solid organ transplant recipients.

\section{Physical protocol measurements and tests}

The standing balance test will be performed with an accelerometer (Axivity, Newcastle, UK), attached to the lower back. The standing balance test has been described in detail previously. ${ }^{29}$ Balance will be evaluated in five different positions: (1) feet together on hard surface, eyes open; (2) feet together on hard surface, eyes closed; (3) feet together on foam surface (Balance Pad Elite; Airex Specialty Foams, Aargau, Switzerland), eyes open; (4) feet together on foam surface, eyes closed; and (5) feet in tandem stance, eyes open. Participants will be asked to have arms crossed on their chest and each position will be tested for $50 \mathrm{~s}$. On failure, recording the time to failure, a second attempt will be performed. In case of non-success at the second attempt, the test will be discontinued.

Endurance will be tested with a $2 M W T .^{30}$ The $2 \mathrm{MWT}$ has been shown to be highly correlated, without compromising validity and reliability, with the 6 min walking test, an important submaximal exercise test. ${ }^{31} 32$ To calculate the distance covered by subjects on the 2MWT, two pylons are set $15 \mathrm{~m}$ apart and subjects are instructed to walk as fast as possible without running, until the investigator commands them to stop. Participants are updated on the remaining time after 1.00 and $1.45 \mathrm{~min}$, and the final $5 \mathrm{~s}$ are indicated by a countdown. The total walking distance in $2 \mathrm{~min}$ is recorded in total metres covered, with the remaining scored in centimetres.

Locomotion, measured as gait speed, will be tested with a $4 M W T$. Gait speed is a simple measure to summarise the overall disease burden and disability. ${ }^{33} 34$ In brief, two pylons will be set $4 \mathrm{~m}$ apart and subjects instructed to walk at a usual pace. Seconds from start to end of the $4 \mathrm{~m}$ will be recorded. The $4 \mathrm{MWT}$ is measured twice after the first trial round.

Manual dexterity will be measured in all transplant recipients using the 9-Hole Peg Test (9-HPT, Sammons Preston Rolyan, Chicago, Illinois). The 9-HPT requires participants to repeatedly place and remove nine pegs into nine holes, one at a time, as quickly as possible, and is considered to be the gold standard metric for manual dexterity.

Functional mobility will be tested in participants using the FTSTS and TUG. The FTSTS is a functional performance measure of leg strength or the force-generating capacity of the muscle by using the body's weight for resistance during functional activities. ${ }^{35}$ The FTSTS will be executed three times after a first trial round. Participants will be instructed to stand up five times as fast as possible, from sitting position with their feet flat on the floor and arms folded across the chest. Measurements start on command, and subsequently the time required to stand up and return sitting is recorded. Time is measured in seconds and this task is repeated five times. ${ }^{36}$

The TUG is a basic test for functional mobility and is based on strength, coordination and balance. ${ }^{37}$ For the test, a pylon and a chair will be put $3 \mathrm{~m}$ apart. The test will be performed four times, with the first round being a trial. Participants are instructed to stand up from the chair without support of the arms, subsequently walk with their normal gait speed around the pylon and go back to the chair to sit down again. In the case where participants use a walking aid in normal day life, the test will be performed with the use of a walking aid. The TUG is measured in seconds, from the moment the participant is instructed to get up until the moment the participant sits down again.

Transplant recipients have an increased susceptibility to develop peripheral neuropathy and tremor, mainly due to the continuous use of immunosuppressive medication, especially calcineurin inhibitors. ${ }^{38} 39$ Therefore, an extensive neurological examination will be performed and will consist of strength testing, classifying polyneuropathy and tremor quantification. Detailed strength testing of different muscle groups (feet flexion/extension, hip flexion, biceps flexion and wrist extension) will be performed with a digital dynamometer (CIT Technics, Haren, The Netherlands). ${ }^{40}$ Hereafter, sensibility tests will be performed using a pin-prick and monofilament pen (Novo Nordisk BV, Alphen aan den Rijn, The Netherlands) on bare skin five times per measurement at the dorsal side of the first phalange of both feet with the subject closing their eyes. On failure of sensibility, the dorsal side of the foot and lower limb will be tested. Proprioception will be measured by moving the first phalange of both feet in dorsal flexion and plantar flexion five times with the participants closing their eyes. On failure, the dorsal side of the foot and the index finger will be measured.

Vibration sense will be measured using a handheld biothesiometer (Biomedical Instrument, Ohio, USA) ${ }^{41}$ The biothesiometer has a rubber tractor that vibrates at $100 \mathrm{~Hz}$ when operating from $50 \mathrm{~Hz}$ mains. In brief, participants will be measured in a supine position on a bed barefoot. The vibrating tractor will be applied bilaterally to four different measurement points of the participants: top of the hallux, forefoot, lateral malleoli and wrist. Before applying the vibrating tractor to the points to be tested, the amplitude of the vibrating tractor is increased from zero to the point where the vibration is perceptible and beyond the threshold to the highest amplitude possible to familiarise participants with the sensation. For the measurement, the participants will be asked to concentrate on the test and report the first sensation of the vibration by saying 'Stop'. Each measurement point is tested twice. If the difference between the first two measurements is greater than $20 \%$, the measurement point is tested a third time.

Prior to tremor quantification, participants will be asked to complete part $\mathrm{C}$ of the Fahn-Tolosa-Marin Tremor Rating Scale, which involves tremor-related functional disability. ${ }^{42}$ The questionnaire involves speaking, bringing liquids to the mouth, eating, hygienic care, dressing, writing, work and household-related tasks. 
The questionnaire uses a 5 -point scale, with ' $0=$ nofunctional ability' and $4=$ 'severe disability, the task cannot be executed'.

To quantify tremor, two accelerometers (UMCG, Groningen, The Netherlands) will be attached to the dorsal side of both hands. The accelerometers will record movement in the coronal, transversal and sagittal planes, as well as linear acceleration and deceleration in both hands continuously, during the measurements. Amplitudes and frequency of these measurements will be recorded on a stand-alone computer. Participants will be asked to assume seven different positions while seated, which are measured for $30 \mathrm{~s}$ each: arms down, wrists extended; arms forward, wrists and fingers relaxed; arms forward, wrists and fingers in 0 position; index fingers pointed towards each other; bilateral finger-nose task; weighted arms down with wrists expanded; and weighted arms forward with wrists and fingers extended.

Finally, participants will be asked for collection of a breath sample in which hydrogen and methane will be measured with the QuinTron BreathTracker (Milwaukee, Wisconsin, USA). ${ }^{43}$ Both hydrogen and methane are exclusively formed by anaerobic fermentation in the gut, and therefore can be used as markers for methanogenic microflora in transplant recipients. ${ }^{44}$

\section{Cognitive protocol measurements and tests}

The Cognitive Screening Test is a Dutch screening test for dementia, measuring orientation in time and place, and memory for common facts. ${ }^{45}$ The questionnaire consists of 20 items (eg, date of birth, name of the reigning monarch, season) and the score is calculated as the total number of questions answered correctly, with a maximum of 20 .

Nederlandse Leestest voor Volwassenen is the Dutch version of the National Adult Reading Test. The participant has to read aloud a list of 50 irregularly spelled words. The total score on the test is converted into an estimation of the premorbid IQ. ${ }^{46}$

The Clock-Drawing Test is a cognitive screening instrument. ${ }^{47}$ Participants are asked to draw a clock and set the time to 'a quarter to two'. A maximum total score of 14 can be achieved.

The 15 Words Test, the Dutch version of the Rey Auditory Verbal Learning Test, measures verbal memory. ${ }^{48}$ In this task a set of 15 unrelated words is presented to the participant, consecutively over five trials. Participants are asked to recall as many words as possible immediately after each trial (immediate recall). The score is the total words recalled in five trials, with a maximum of 75 . After $20 \mathrm{~min}$, participants are asked to recall as many of the 15 words as possible (delayed recall). Additionally, a recognition task will be performed. Participants are presented with a list of 30 words and are asked which words they recognise from the list they have been presented before.

Digit Span is a subtest of the Wechsler Adult Intelligence Scale IV. ${ }^{49}$ This subtest consists of two tasks, the Digit Span Forward and the Digit Span Backward. The Digit
Span Forward is a task for immediate auditory memory span. In this task, participants are asked to repeat a series of numbers in the same order as the examiner did. The Digit Span Backward measures working memory. Participants have to repeat the presented numbers in reversed order. The score is the total strings repeated, with a maximum of 32.

The Word Fluency Test, a subtest of the Groninger Intelligentie Test 2, is a verbal task measuring semantic memory ${ }^{50}$ Participants are asked to name as many words within a certain category within $1 \mathrm{~min}$. The total score per category (respectively animals and professions) was calculated.

The Controlled Oral Word Association Test is a verbal task measuring executive control. ${ }^{51}$ Participants have to name as many words as possible that start with a specific letter within $1 \mathrm{~min}$. In the meantime, participants have to comply to several rules that are given beforehand. The total scores from three different starting letters (D-A-T) were calculated.

The Symbol Digit Modalities Test measures psychomotor speed. ${ }^{52}$ The test consists of matching symbols and numbers as fast as possible in $90 \mathrm{~s}$. The total score of correct matches is calculated.

The Trail Making Test (TMT) consists of two parts: Trail Making Test-A (TMT-A) and Trail Making Test-B (TMT-B). TMT-A is a measure of attention and information processing speed. This task involves connecting 25 numbers in ascending order, as quickly as possible. The TMT-B is a measure of divided attention and cognitive flexibility. In this condition, numbers as well as letters have to be connected in ascending order, alternating between numbers and letters (1-A-2-B- and so on). Both parts of the test are timed to completion (number of seconds).

The Key Search Test is a subtest of the Behavioural Assessment of the Dysexecutive Syndrome and assesses the ability to plan and monitor progress. Participants are presented with a square which represents a field in which 'keys have been lost'. Participants must show how they would search the field to find the keys. Searching strategy is scored by means of functionality and a maximum total score of 16 can be achieved.

\section{Outcomes}

The primary outcomes of the TransplantLines study are all-cause mortality and graft failure, which are defined as death due to failure of the transplanted organ, return to organ replacement therapy or retransplantation. The secondary outcomes will be cause-specific mortality, cause-specific graft failure and rejection. The tertiary outcomes will be other health problems, including diabetes, obesity, hypertension, hypercholesterolaemia and cardiovascular disease, and disturbances that relate to quality of life, for example, physical and psychological functioning, quality of sleep, and neurological problems such as tremor and polyneuropathy.

The TransplantLines biobank study aims to identify the risk factors for health problems and patient-centred 
outcomes (eg, adverse drug events, lifestyle, quality of life, social participation, physical and cognitive functioning). Due to the nature of the biobank, not all research questions are predefined and will arise during the course of inclusion. In contrast to many other studies, TransplantLines also aims to identify and ameliorate complaints experienced by transplant recipients, such as tremors and diarrhoea, which to date have largely been overlooked by clinicians.

\section{Data management}

Analysis, and access to data and samples

Data will be recorded digitally in an electronic case report form in a certified Electronic Data Capture and Clinical Data Management System (Utopia Data Management System V.1.13.6, Research Data Support, UMCG). Data entry is performed by the trained investigators. The trained investigator who performed assessments at the study visit of a participant is responsible for data entry of that participant. All data are later checked again by the trained investigators and are subsequently stored anonymously in a secured electronic environment. The TransplantLines database will be linked to registries and databases of the Dutch Health Database, Netherlands Comprehensive Cancer Organisation, Central Bureau of Statistics, InterAction Database, Dutch Nephrology Registration/Registration Renal Replacement Therapy (Nefrovisie, Renine), Nationwide Network and Registry of Histo- and Cytopathology in the Netherlands (PALGA), National Organ Transplant Registry, PHARMO Institute for Drug Outcomes Research, Routine Outcome Monitoring, and the Dutch Institute of Clinical Auditing database through a generic layer. A data management board will be formed to maintain data infrastructure, construct material transfer agreements, and to govern use of the TransplantLines biobank and database. Extractions from TransplantLines database will be performed using a retrieval suite in Utopia software package only after approval of the data management board. Data will always be extracted anonymously. SPSS Statistics V.23, R V.3.2.3 (CRAN, Vienna, Austria), STATA V.14.1 or a similar statistical package will be used for analysis. Data collection and management is performed in accordance with the Handbook for Adequate Natural Data Stewardship (Netherlands Federation of University Medical Centers, 2017). A team consisting of medical doctors of the different fields involved, called Research Team TransplantLines, is installed to decide and prioritise who will get access to the samples and data of the TransplantLines biobank and cohort study. Use of samples and data can be requested by internal and external researchers against a reasonable fee. All samples are stored at $-80^{\circ} \mathrm{C}$ and access are logged in a linked database. The logging system also provides for registration of multiple access and the number of freezethaw cycles that the samples have undergone. Multiple access to samples is possible, but for each specific project a new request needs to be performed and approved by the Research Team TransplantLines. The data available from the assays performed on the provided samples will be linked to the TransplantLines database and be made available to researchers in the certified Electronic Data Capture and Clinical Data Management System, which will allow for evaluation and statistical analyses. This environment will also monitor and log data handling and store the results of the analyses.

\section{Missing data handling}

Concerning treatment of missing data and inability to generate data from missing samples, we will apply statistical methods using maximum likelihood and multiple imputation, which are now standard for dealing with loss of participants and missing data. ${ }^{53}$ These methods provide more consistent and efficient estimates of population parameters than methods relying on complete cases, mean imputation, last observation carried forward or single-imputation regression methods ${ }^{53-56}$ As advised in authoritative reports, these analyses will be complemented with sensitivity analyses to assess robustness of findings. ${ }^{57-59}$

\section{DISCUSSION}

The TransplantLines prospective cohort study seeks to identify the risk factors for the development of long-term health problems after transplantation, and ultimately to develop new and innovative interventions to improve graft survival, patient survival and quality of life after transplantation. The TransplantLines biobank will encompass all solid organ transplantations and living organ donors. It will consist of follow-up data from all fields that are involved in organ transplantation: internal medicine, surgery, gastroenterology, hepatology, pulmonology, cardiology, dermatology, neurology, occupational medicine, children's medicine, (neuro)psychology, physiotherapy and social work.

Although short-term transplant outcomes have improved in the last decades, graft and recipient life expectancy remains limited. In the TransplantLines study, data and samples will be collected before, during and after transplantation to gather further insight into the impact of transplantation on transplant recipients. In addition, we aim to pre-emptively detect those transplant recipients who are at increased risk to develop graft failure or health problems. By investigating a wide range of clinical, social/psychological and biochemical parameters, this study aims to contribute to increased transplant survival and patient survival, but also to an increased quality of life and a more patient-centred approach to transplant care.

Our study has strengths and limitations. The major strengths of this study are the collection of extensive data on a myriad topics related to transplantation, the inclusion of all types of solid organ transplant recipients and living organ donors, and a study with a long follow-up to assess many relevant clinical outcomes. 
The limitations of the current study are that it is a single-centre study and that residual confounding cannot be excluded in analyses due to its observational design. Another limitation is that transplant recipients with limited language skills and/or poor comprehension are excluded because these patients are likely those who are at higher risk of poor compliance and high risk of social behaviour, which would possibly have worse outcomes. A further limitation is that our infrequent collection of biobank samples may limit utility in detecting biomarkers for routine monitoring of transplant health, and detection of suitable biomarkers may only be possible if sampling happens to fall near the time of a clinical event. It may be considered a strength that in addition to taking samples at fixed timepoints, we also take samples when biopsies are performed, both at the time of protocol biopsies and at the time of biopsies taken at clinical indication, usually because of worsening of transplant function, with suspicion of acute or chronic rejection.

TransplantLines may serve as a basis for hypothesis-generating studies that yield insights into a wide range of clinical, social/psychological and biochemical parameters in solid organ transplant recipients as well as living donors. Biomarkers may be identified to develop more individualised immunosuppressive treatment. This will lead to novel clinical trials in transplantation and patient-tailored approaches for new treatment options. Furthermore, the results of TransplantLines may serve to identify new modifiable risk factors and lifestyle factors in transplantation. Ultimately, this information will likely contribute to a more individualised treatment for transplant patients and improved living donor screening and follow-up. Thereby we aim to qualitatively and quantitatively improve outcomes after transplantation.

\section{STUDY STATUS}

Data collection is in progress.

\section{Author affiliations}

${ }^{1}$ Division of Nephrology, Department of Internal Medicine, University of Groningen, University Medical Center Groningen, Groningen, The Netherlands

${ }^{2}$ Department of Neuropsychology, University of Groningen, University Medical Center Groningen, Groningen, The Netherlands

${ }^{3}$ Groningen Transplant Center, University of Groningen, University Medical Center Groningen, Groningen, The Netherlands

${ }^{4}$ Department of Dermatology, University of Groningen, University Medical Center Groningen, Groningen, The Netherlands

${ }^{5}$ Department of Pediatrics, University of Groningen, University Medical Center Groningen, Groningen, The Netherlands

${ }^{6}$ Department of Surgery, University of Groningen, University Medical Center Groningen, Groningen, The Netherlands

${ }^{7}$ Department of Neurology, University of Groningen, University Medical Center Groningen, Groningen, The Netherlands

${ }^{8}$ Department of Cardiology, University of Groningen, University Medical Center Groningen, Groningen, The Netherlands

${ }^{9}$ Department of Pulmonary Diseases and Tuberculosis, University of Groningen, University Medical Center Groningen, Groningen, The Netherlands

${ }^{10}$ Department of Gastroenterology and Hepatology, University of Groningen, University Medical Center Groningen, Groningen, The Netherlands
Acknowledgements We thank Professor John Mathers and Dr José Lara from Newcastle University for aid in setting up a test package for the TransplantLines study based on the Healthy Ageing Phenotype measurements.

Collaborators Martin H De Borst, Margriet FC De Jong, Jan Stephan F Sanders, Gerjan Navis (Division of Nephrology, University of Groningen, University Medical Center Groningen, Groningen, The Netherlands); Jan Willem J Elting, Marina AJ Tijssen (Department of Neurology, University of Groningen, University Medical Center Groningen, Groningen, The Netherlands); Marieke T de Boer (Department of Surgery, University of Groningen, University Medical Center Groningen, Groningen, The Netherlands); Adelita V Ranchor (Department of Health Psychology, University of Groningen, University Medical Center Groningen, Groningen, The Netherlands); Ilja M Nolte, Rob J Bieringa, Paul Koenes (Department of Epidemiology, University of Groningen, University Medical Center Groningen, Groningen, The Netherlands); Wim van der Bij (Department of Pulmonary Diseases and Tuberculosis, University of Groningen, University Medical Center Groningen, Groningen, The Netherlands).

Contributors MFE designed the study, conducts the study for transplant recipients, wrote the manuscript and is responsible for final content of the manuscript. AWG-N conducts the study for transplant recipients and revised the manuscript. MvL conducts the study for living donors and wrote the part of living donors in the manuscript. ALZ conducts the study for transplant recipients, living donors and wrote the cognitive protocol. RMD, SPS, TJK and MCJO conduct the study for transplant recipients. NRH conducts the study for living donors. AMB, MJS, CA, ER, JMS, FAJAB, RAP, SPB, GD, RJP, HGDL, KD, EAMV and VEdM gave substantial input from their field of knowledge when designing the study and revised the manuscript. RAP is responsible for kidney biobank sampling and logistics. HB conducts the study and revised the manuscript. SJLB designed the study, is the principal investigator and project leader, and takes full responsibility for the whole study. All authors approved the final version of the manuscript and agree to be accountable for all aspects of the submitted work. All collaborators have revised the manuscript and approved the final version of the manuscript.

Funding This work was supported by a grant from Astellas BV.

Competing interests None declared.

Patient consent for publication Obtained.

Ethics approval The study protocol has been approved by the Institutional Review Board (METc 2014/077) (METc UMCG), adheres to the UMCG Biobank Regulation, and is in accordance with the WMA Declaration of Helsinki and the Declaration of Istanbul.

Provenance and peer review Not commissioned; externally peer reviewed.

Data sharing statement Unpublished data are primarily intended for researchers at the University Medical Center Groningen and involved in the TransplantLines study. Access to data from external parties will be evaluated in a central meeting of the Research Team TransplantLines. Use of samples and data can be requested by internal and external researchers against a reasonable fee.

Open access This is an open access article distributed in accordance with the Creative Commons Attribution Non Commercial (CC BY-NC 4.0) license, which permits others to distribute, remix, adapt, build upon this work non-commercially, and license their derivative works on different terms, provided the original work is properly cited, appropriate credit is given, any changes made indicated, and the use is non-commercial. See: http://creativecommons.org/licenses/by-nc/4.0/.

\section{REFERENCES}

1. Kirk R, Dipchand AI, Edwards LB, et al. The registry of the international society for heart and lung transplantation: fifteenth pediatric heart transplantation report-2012. J Heart Lung Transplant 2012;31:1065-72.

2. Lamb KE, Lodhi S, Meier-Kriesche HU. Long-term renal allograft survival in the United States: a critical reappraisal. Am J Transplant 2011;11:450-62.

3. Lodhi SA, Lamb KE, Meier-Kriesche HU. Solid organ allograft survival improvement in the United States: the long-term does not mirror the dramatic short-term success. Am J Transplant 2011;11:1226-35.

4. Dharnidharka VR, Lamb KE, Zheng J, et al. Lack of significant improvements in long-term allograft survival in pediatric solid organ transplantation: A US national registry analysis. Pediatr Transplant 2015;19:477-83.

5. Hariharan S, Johnson CP, Bresnahan BA, et al. Improved graft survival after renal transplantation in the United States, 1988 to 1996. N Engl J Med 2000;342:605-12. 
6. Aasebø W, Homb-Vesteraas NA, Hartmann A, et al. Life situation and quality of life in young adult kidney transplant recipients. Nephrol Dial Transplant 2009;24:304-8.

7. Kugler C, Fischer S, Gottlieb J, et al. Symptom experience after lung transplantation: impact on quality of life and adherence. Clin Transplant 2007;21:590-6.

8. Matas AJ, McHugh L, Payne WD, et al. Long-term quality of life after kidney and simultaneous pancreas-kidney transplantation. Clin Transplant 1998;12:233-42.

9. Morath $\mathrm{C}$, Mueller M, Goldschmidt $\mathrm{H}$, et al. Malignancy in renal transplantation. J Am Soc Nephrol 2004;15:1582-8.

10. Rodrigo E, Fernández-Fresnedo G, Valero R, et al. New-onset diabetes after kidney transplantation: risk factors. J Am Soc Nephrol 2006;17:S291-S295.

11. Jezior D, Krajewska M, Madziarska K, et al. Posttransplant overweight and obesity: myth or reality? Transplant Proc 2007;39:2772-5.

12. Held PJ, McCormick F, Ojo A, et al. A cost-benefit analysis of government compensation of kidney donors. Am J Transplant 2016;16:877-85.

13. Mjøen G, Hallan S, Hartmann A, et al. Long-term risks for kidney donors. Kidney Int 2014;86:162-7.

14. Muzaale AD, Massie AB, Wang MC, et al. Risk of end-stage renal disease following live kidney donation. JAMA 2014;311:579-86.

15. Massie AB, Leanza J, Fahmy LM, et al. A risk index for living donor kidney transplantation. Am J Transplant 2016;16:2077-84.

16. Reese PP, Boudville N, Garg AX. Living kidney donation: outcomes, ethics, and uncertainty. Lancet 2015;385:2003-13.

17. Miller CM, Durand F, Heimbach JK, et al. The international liver transplant society guideline on living liver donation. Transplantation 2016;100:1238-43.

18. Trotter JF. Selection of donors and recipients for living donor liver transplantation. Liver Transp/ 2000;6:s52-s58.

19. Angst F, Drerup S, Werle S, et al. Prediction of grip and key pinch strength in 978 healthy subjects. BMC Musculoskelet Disord 2010;11:94

20. Trampisch US, Franke J, Jedamzik N, et al. Optimal Jamar dynamometer handle position to assess maximal isometric hand grip strength in epidemiological studies. $J$ Hand Surg Am 2012;37:2368-73.

21. Drew CD, Hughes DT. Characteristics of the Vitalograph spirometer. Thorax 1969;24:703-6.

22. Kyle UG, Bosaeus I, De Lorenzo AD, et al. Bioelectrical impedance analysis-part I: review of principles and methods. Clin Nutr 2004;23:1226-43.

23. Mulder DJ, Water TV, Lutgers HL, et al. Skin autofluorescence, a novel marker for glycemic and oxidative stress-derived advanced glycation endproducts: an overview of current clinical studies, evidence, and limitations. Diabetes Technol Ther 2006;8:523-35.

24. Mclntyre NJ, Fluck RJ, McIntyre CW, et al. Skin autofluorescence and the association with renal and cardiovascular risk factors in chronic kidney disease stage 3. Clin J Am Soc Nephrol 2011;6:2356-63.

25. O'Reilly Zwald F, Brown M. Skin cancer in solid organ transplant recipients: advances in therapy and management: part I. Epidemiology of skin cancer in solid organ transplant recipients. $J$ Am Acad Dermatol 2011;65:253-61. quiz 262.

26. Fitzpatrick TB. The validity and practicality of sun-reactive skin types I through VI. Arch Dermatol 1988;124:869-71.

27. Rockwood K, Song X, MacKnight C, et al. A global clinical measure of fitness and frailty in elderly people. CMAJ 2005;173:489-95.

28. Ottery FD. Definition of standardized nutritional assessment and interventional pathways in oncology. Nutrition 1996;12:S15-S19.

29. Reuben DB, Magasi S, McCreath HE, et al. Motor assessment using the NIH Toolbox. Neurology 2013;80:S65-S75.

30. Bohannon RW, Wang YC, Gershon RC. Two-minute walk test performance by adults 18 to 85 years: normative values, reliability, and responsiveness. Arch Phys Med Rehabil 2015;96:472-7.

31. ATS Committee on Proficiency Standards for Clinical Pulmonary Function Laboratories. ATS statement: guidelines for the six-minute walk test. Am J Respir Crit Care Med 2002;166:111-7.

32. Butland RJ, Pang J, Gross ER, et al. Two-, six-, and 12-minute walking tests in respiratory disease. Br Med J 1982;284:1607-8.

33. Studenski S, Perera S, Patel K, et al. Gait speed and survival in older adults. JAMA 2011;305:50-8.

34. Perera S, Patel KV, Rosano C, et al. Gait speed predicts incident disability: a pooled analysis. J Gerontol A Biol Sci Med Sci 2016;71:63-71.

35. Bohannon RW, Barreca SR, Shove ME, et al. Documentation of daily sit-to-stands performed by community-dwelling adults. Physiother Theory Pract 2008;24:437-42.
36. Bohannon RW. Test-retest reliability of the five-repetition sit-to-stand test: a systematic review of the literature involving adults. J Strength Cond Res 2011;25:3205-7.

37. Kwan MM, Lin SI, Chen $\mathrm{CH}$, et al. Sensorimotor function, balance abilities and pain influence Timed Up and Go performance in older community-living people. Aging Clin Exp Res 2011;23:196-201.

38. Arnold R, Pussell BA, Pianta TJ, et al. Association between calcineurin inhibitor treatment and peripheral nerve dysfunction in renal transplant recipients. Am J Transplant 2013;13:2426-32.

39. Langone A, Steinberg SM, Gedaly R, et al. Switching STudy of Kidney TRansplant PAtients with Tremor to LCP-TacrO (STRATO): an open-label, multicenter, prospective phase 3b study. Clin Transplant 2015;29:796-805.

40. van der Ploeg RJ, Fidler V, Oosterhuis HJ. Hand-held myometry: reference values. J Neurol Neurosurg Psychiatry 1991:54:244-7.

41. Frenette B, Mergler D, Ferraris J. Measurement precision of a portable instrument to assess vibrotactile perception threshold. Eur $\mathrm{J}$ Appl Physiol Occup Physiol 1990;61:386-91.

42. Elble R, Bain P, Forjaz MJ, et al. Task force report: scales for screening and evaluating tremor: critique and recommendations. Mov Disord 2013;28:1793-800.

43. Tap J, Derrien M, Törnblom H, et al. Identification of an intestinal microbiota signature associated with severity of irritable bowel syndrome. Gastroenterology 2017;152:111-.

44. Sahakian AB, Jee SR, Pimentel M. Methane and the gastrointestinal tract. Dig Dis Sci 2010;55:2135-43.

45. Ad G, Deelman BG. Cognitieve screening test. Handleiding. Lisse: Swets and Zeitlinger, 1991.

46. Schmand B, Lindeboom J, van Harskamp F. Nederlandse leestest voor volwassenen: handleiding (NLV). Amsterdam: Pearson Assessment and Information B.V, 1992.

47. Shulman KI, Shedletsky R, Silver IL. The challenge of time: Clockdrawing and cognitive function in the elderly. Int $J$ Geriatr Psychiatry 1986;1:135-40.

48. Saan R, Deelman B. De 15-Woorden Tests A en B. (Een voorlopige handleiding). University Medical Center Groningen, department of Neuropsychology: Groningen, 1986.

49. Wechsler D. WAIS-IV-NL: Wechsler Adult Intelligence Scale - Fourth Edition - Nederlandstalige bewerking: Technische handleiding en Afname en scoringshandleiding. Amsterdam: Pearson Assessment and Information B.V, 2012.

50. Luteijn F, Barelds D. GIT2: Groninger Intelligentie Test 2. Amsterdam: Pearson Assessment and Information B.V, 2004.

51. Schmand B, Groenink SC, den Dungen M. Letterfluency: psychometrische eigenschappen en Nederlandse normen. Tijdschr Gerontol Geriatr 2008;39:64-74

52. Smith A. Symbol digits modalities test Los Angeles: Western Psychological Services. Los Angeles: Western Psychological Services, 1968:83-91.

53. Christensen H, Mackinnon A, Jorm AF, et al. The Canberra longitudinal study: design, aims, methodology, outcomes and recent empirical investigations. Aging, Neuropsychology, and Cognition 2004;11:169-95

54. Woolley SB, Cardoni AA, Goethe JW. Last-observation-carriedforward imputation method in clinical efficacy trials: review of 352 antidepressant studies. Pharmacotherapy 2009;29:1408-16.

55. Schafer JL, Graham JW. Missing data: our view of the state of the art. Psychol Methods 2002;7:147-77.

56. Graham JW. Missing data analysis: making it work in the real world. Annu Rev Psychol 2009;60:549-76.

57. Little RJ, D'Agostino R, Cohen ML, et al. The prevention and treatment of missing data in clinical trials. $N$ Engl $J$ Med 2012;367:1355-60.

58. Lee KJ, Simpson JA. Introduction to multiple imputation for dealing with missing data. Respirology 2014;19:162-7.

59. Sainani KL. Dealing with missing data. Pm R 2015;7:990-4.

60. Hoeymans $\mathrm{N}$, van Lindert $\mathrm{H}$, Westert GP. The health status of the Dutch population as assessed by the EQ-6D. Qual Life Res 2005;14:655-63.

61. Reips UD, Funke F. Interval-level measurement with visual analogue scales in Internet-based research: VAS Generator. Behav Res Methods 2008;40:699-704.

62. Ware JE, Sherbourne CD. The MOS 36-item short-form health survey (SF-36). I. Conceptual framework and item selection. Med Care 1992;30:473-83.

63. Wendel-Vos GC, Schuit AJ, Saris WH, et al. Reproducibility and relative validity of the short questionnaire to assess health-enhancing physical activity. J Clin Epidemiol 2003;56:1163-9.

64. Schäfer-Keller P, Steiger J, Bock A, et al. Diagnostic accuracy of measurement methods to assess non-adherence to immunosuppressive drugs in kidney transplant recipients. $A m \mathrm{~J}$ Transplant 2008;8:616-26. 
65. Dobbels $F$, Moons $\mathrm{P}$, Abraham I, et al. Measuring symptom experience of side-effects of immunosuppressive drugs: the Modified Transplant Symptom Occurrence and Distress Scale. Transpl Int 2008;21:764-73.

66. Beurskens AJ, Bültmann U, Kant I, et al. Fatigue among working people: validity of a questionnaire measure. Occup Environ Med 2000;57:353-7.

67. Buysse DJ, Reynolds CF, Monk TH, et al. The Pittsburgh Sleep Quality Index: a new instrument for psychiatric practice and research. Psychiatry Res 1989;28:193-213.

68. Marteau TM, Bekker H. The development of a six-item short-form of the state scale of the Spielberger State-Trait Anxiety Inventory (STAI). Br J Clin Psychol 1992;31(Pt 3):301-6.

69. Kroenke K, Spitzer RL, Williams JB. The PHQ-9: validity of a brief depression severity measure. J Gen Intern Med 2001;16:606-13.

70. Gillanders DT, Bolderston H, Bond FW, et al. The development and initial validation of the cognitive fusion questionnaire. Behav Ther 2014;45:83-101.
71. Topp CW, Østergaard SD, Søndergaard S, et al. The WHO-5 Well-Being Index: a systematic review of the literature. Psychother Psychosom 2015;84:167-76.

72. Ziegelmann JP, Griva K, Hankins M, et al. The Transplant Effects Questionnaire (TxEQ): the development of a questionnaire for assessing the multidimensional outcome of organ transplantation - example of end stage renal disease (ESRD). Br J Health Psychol 2002;7:393-408.

73. Pearlin LI, Schooler C. The structure of coping. $J$ Health Soc Behav 1978;19:2-21.

74. Abma FI, Amick BC, Brouwer S, et al. The cross-cultural adaptation of the work role functioning questionnaire to Dutch. Work 2012:43:203-10.

75. Longstreth GF, Thompson WG, Chey WD, et al. Functional bowel disorders. Gastroenterology 2006;130:1480-91.

76. Willett WC, Sampson L, Stampfer MJ, et al. Reproducibility and validity of a semiquantitative food frequency questionnaire. $A m \mathrm{~J}$ Epidemiol 1985;122:51-65. 